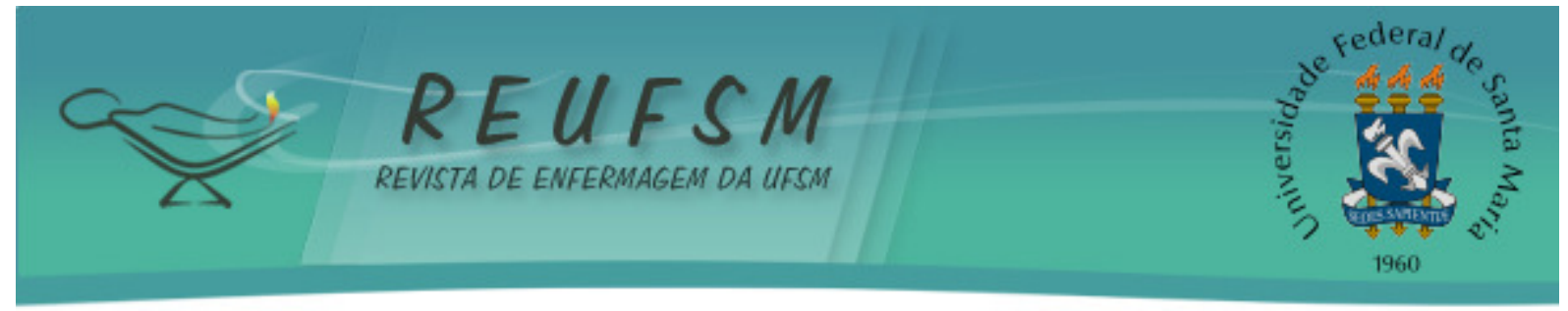

ARTIGO ORIGINAL

\title{
CUIDADO DE PRESERVAÇÃO DESENVOLVIDO POR FAMILIARES/CUIDADORES DE CRIANÇA COM ASMA
}

\section{PRESERVATION CARE DEVELOPED BY FAMILYICAREGIVERS OF CHILDREN WITH ASTHMA CUIDADOS DE PRESERVACIÓN DESARROLLADOS POR FAMILIARES/CUIDADORES DE NIÑOS CON ASMA}

Doi: $10.5902 / 2179769215853$

Camila Fernandes Wild ${ }^{1}$ Andressa da Silveira ${ }^{2}$

RESUMO: Objetivo: apresentar as estratégias de cuidado dos familiares/cuidadores de crianças com asma no domicílio. Método: pesquisa de caráter exploratório e descritivo, com abordagem qualitativa. As participantes da pesquisa foram 15 familiares de crianças com asma de um serviço público de saúde. Para a coleta de dados utilizou-se a entrevista semiestruturada com amostragem por saturação, e, posteriormente, aplicou-se a análise de discurso na corrente francesa. Resultados: as familiares realizam um cuidado de preservação da saúde da criança, o que faz com que em alguns momentos as cuidadoras abneguem de sua vida social e pessoal devido à preocupação com o estado de saúde da criança e com estratégias para a manutenção de sua sobrevivência. Conclusão: o cuidado domiciliar vai além do tratamento, trata-se da conservação da vida da criança, ou seja, um cuidado de preservação, predominantemente desenvolvido pelas mulheres de cada família e está pautado em estratégias e recursos para a manutenção da sobrevivência dessas crianças.

Descritores: Família; Asma; Saúde da criança; Enfermagem.

ABSTRACT: Aim: to present care strategies used by family members/caregivers of children with asthma in home space. Method: exploratory and descriptive research, with a qualitative approach. The participants were 15 parents of children with asthma from a public health service. To collect data, there were used semi-structured interviews with a saturation sample, subsequently applied to the analysis of discourse in the French stream. Results: the family held a care of preserving the health of the child, which makes caregivers recuse social and personal, in some moments, due to the concern about the situation of the child health and strategies for maintaining their survival. Conclusion: home care goes beyond treatment, it is the preservation of the child's life, that is, a preservation care. Care is predominantly developed by the women of each family and is guided by strategies and resources for the maintenance of survival of these patients.

Descriptors: Family; Asthma; Child health; Nursing.

RESUMEN: Objetivo: presentar las estrategias de atención utilizada por familiares/cuidadores de los niños con asma en el espacio doméstico. Método: Estudio exploratorio y descriptivo, con enfoque cualitativo. Los participantes de la investigación fueron 15 familiares de niños con asma de un servicio de salud pública. En la recogida de los datos se utilizó la entrevista semi-estructurada con muestreo de saturación y posterior

\footnotetext{
${ }^{1}$ Enfermeira. Mestranda em Enfermagem pela Universidade Federal de Santa Maria. Santa Maria, RS, Brasil. Email: camilinhah_wild@hotmail.com

${ }^{2}$ Enfermeira. Doutoranda em Enfermagem pela Universidade Federal de Santa Maria. Professora Assistente do Curso de Graduação em Enfermagem na Universidade Federal do Pampa. Campus Uruguaiana. Santa Maria, RS, Brasil. E-mail: andressadasilveira@gmail.com
} 


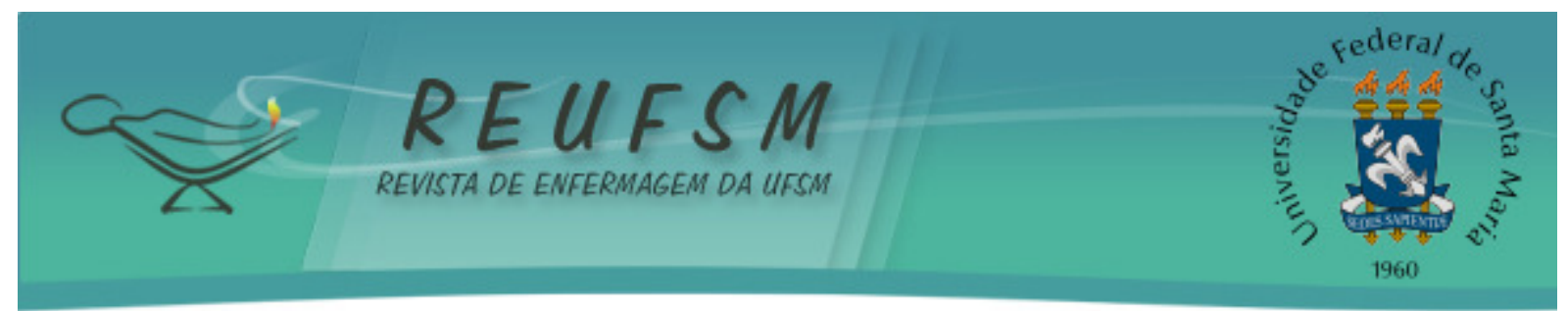

aplicación del análisis del discurso en cadena francesa. Resultados: los cuidadores realizan una preservación del cuidado de la salud del niño, que resulta en la recusa de su vida social debido a la preocupación del estado de salud del niño y de estrategias para su supervivencia. Conclusión: el cuidado en el hogar va más allá del tratamiento, es la preservación de la vida del asmático, un cuidado desarrollado por las mujeres de la familia, que se guíam por estrategias y recursos de manutención y supervivencia de estos niños.

Descriptores: Familia; Asma; Salud infantil; Enfermería.

\section{INTRODUÇÃO}

A asma é uma doença complexa que provoca alterações no desenvolvimento pessoal da criança e na dinâmica familiar e social. A doença apresenta-se de formas distintas na sociedade segundo suas especificidades culturais, sociais e históricas, trazendo uma série de significados, além dos conhecimentos científicos dos profissionais da saúde. ${ }^{1}$

Ademais, a asma é considerada a doença crônica mais comum na infância, gerando absenteísmo escolar e um importante impacto na economia e na qualidade de vida da criança. Contabiliza-se cerca de 250 mil internações ao ano em decorrência da asma. Esta representa a terceira causa de internações entre crianças e adultos jovens na atualidade. ${ }^{2}$

A experiência de viver com asma envolve aqueles que convivem com a criança, especialmente os familiares. Estes são impactados tanto pelas transformações do dia a dia, quanto pela clínica da doença, resultando em profundas modificações na dinâmica familiar..$^{3-4}$

Os familiares da criança com asma defrontam-se com demandas de cuidados intensos, que exigem constante vigilância e envolvem procedimentos e orientações de enfermagem que a família precisa incluir no seu dia a dia. Ressalta-se que pouco se sabe sobre a rede familiar de cuidados, sua forma de organização e sobre os recursos que possuem para conduzi-los no domicílio. ${ }^{5}$

Cada núcleo familiar é singular e o enfrentamento das adversidades oriundas do percurso da asma influencia positivamente na adaptação e, consequentemente, na resposta da família e da criança ao tratamento, resultando na melhora da qualidade de vida. ${ }^{3}$

Dessa forma, a família da criança é de extrema importância para a continuidade do cuidado domiciliar. Cuidar, na perspectiva de inclusão da família, é propor espaços para a dialogicidade entre o familiar/cuidador e os profissionais de saúde, a fim de que o domicílio seja uma extensão para as práticas de cuidado. ${ }^{6}$

0 presente estudo justifica-se diante da necessidade de enfatizar a singularidade do cuidado domiciliar desenvolvido pelos familiares/cuidadores da criança com asma, pela escassez de produções científicas que retratam esta temática, embora a asma tenha números consideráveis no cenário nacional. Além disso, acredita-se que a partir dos resultados dessa pesquisa é possível reconhecer o espaço domiciliar e suas possibilidades para o cuidado da criança com asma.

Frente ao exposto, o estudo tem como questão de pesquisa: quais as estratégias de cuidado utilizadas por familares/cuidadores de criança com asma?

0 artigo objetiva apresentar as estratégias de cuidado dos familiares/cuidadores de crianças com asma no domicílio.

\section{MÉTODO}

Trata-se de um estudo de caráter exploratório e descritivo, com abordagem qualitativa. Para a coleta de dados elegeu-se a entrevista semiestruturada, na qual o entrevistado tem a possibilidade de descrever o tema questionado, sem ter de preocupar- 


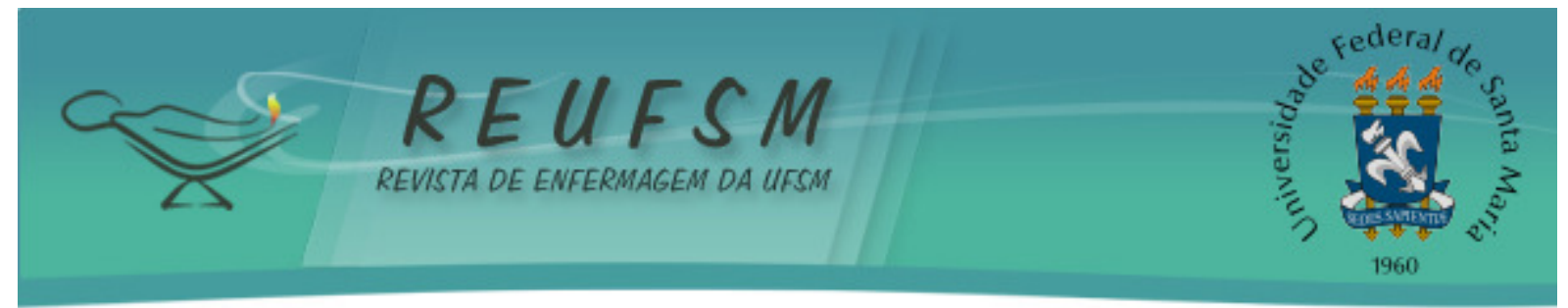

se com as respostas e as condições fixadas pelo pesquisador, pois se trata de perguntas fechadas ou abertas, em que maior ênfase se dá nas interações.

A pesquisa foi desenvolvida no primeiro semestre de 2014. O cenário eleito para o estudo foi uma Policlínica Infantil, situada no município de Uruguaiana, Rio Grande do Sul. O serviço caracteriza-se por ser um centro de referência público no atendimento às crianças com asma na fronteira oeste do Estado e recebe em média 400 crianças ao mês.

Foram inclusos no estudo familiares/cuidadores que desempenhavam cuidados no ambiente domiciliar à criança com asma, e que frequentassem a policlínica infantil no acompanhamento da criança. Os participantes do estudo foram 15 familiares/cuidadores de crianças com asma, que fazem parte do Programa Infantil de Prevenção da Asma (PIPA). Foram identificados no estudo pelo codinome FC relativo à familiar/cuidador seguindo uma numeração ordinal aleatória.

Foi utilizada a amostragem por saturação, operacionalmente definida como a suspensão de inclusão de novos participantes quando os dados obtidos passam a apresentar, na avaliação do pesquisador, uma certa redundância ou repetição, não sendo considerado relevante persistir na coleta de dados. ${ }^{8}$

Após a transcrição das enunciações, utilizou-se a Análise de Discurso (AD) na corrente francesa. $O$ objetivo da $A D$ é realizar uma reflexão geral sobre a significação dos textos produzidos para compreender o modo de funcionamento, os princípios de organização e as formas de produção de seus sentidos. ${ }^{9}$

Esta análise serve para dar expressão ao texto, possibilitando ao leitor compreender as falas dos participantes. Para tanto, inclui-se materialidade linguística aos discursos por meio de sinais ortográficos como: / Pausa reflexiva curta; // Pausa reflexiva longa; /// Pausa reflexiva muito longa; ... Pensamento incompleto; \# Interrupção da fala do sujeito; [ ] Explicação/ Correção da palavra ou frase incompleta; [...] Recorte de um trecho do discurso; “..." aspas duplas indicam a fala ou texto de outrem. Posteriormente aplicou-se as ferramentas analíticas como a metáfora, paráfrase e polissemia.

A pesquisa teve início após a aprovação pelo Comitê de Ética em Pesquisa (CEP) da Universidade Federal do Pampa (UNIPAMPA) sob o número 498.734. Além disto, seguiu-se os padrões éticos estabelecidos pela Resolução 466/2012. ${ }^{10}$

\section{RESULTADOS}

Dentre os participantes da pesquisa, havia 11 mães, três avós e uma bisavó; $54 \%$ tinham entre 30 e 40 anos, 33\% acima de 40 anos e 13\% entre 20 e 30 anos. Ressalta-se que $53 \%$ viviam abaixo da linha da pobreza, com uma renda de até um salário mínimo.

As familiares/cuidadoras relataram o manejo e os recursos utilizados no domicílio para evitar as crises asmáticas. A necessidade do cuidado incessante faz com que a dinâmica familiar seja reorganizada para buscar alternativas para evitar os agravos à saúde da criança. Para mantê-las livres de riscos, as familiares/cuidadoras buscam diversas estratégias, conforme as enunciações a seguir:

tem nebulização, tem os remédios né!? Primeiro a gente faz o que tem que fazer. Depois se apurar [metaforicamente falando] muito a crise [de asma] e a febre aumentar, Aí tem que levar... [levar para o hospital.] (FC1)

[...] Eu deixo a casa bem ventilada [...]! Ela usa dois tipos de remédios de uso contínuo, eu dou a metade de um comprido todos os dias de noite pra ela, e coloco spray no nariz dela... todos os dias de noite eu tenho que fazer isso pra poder controlar a asma! (FC2) 


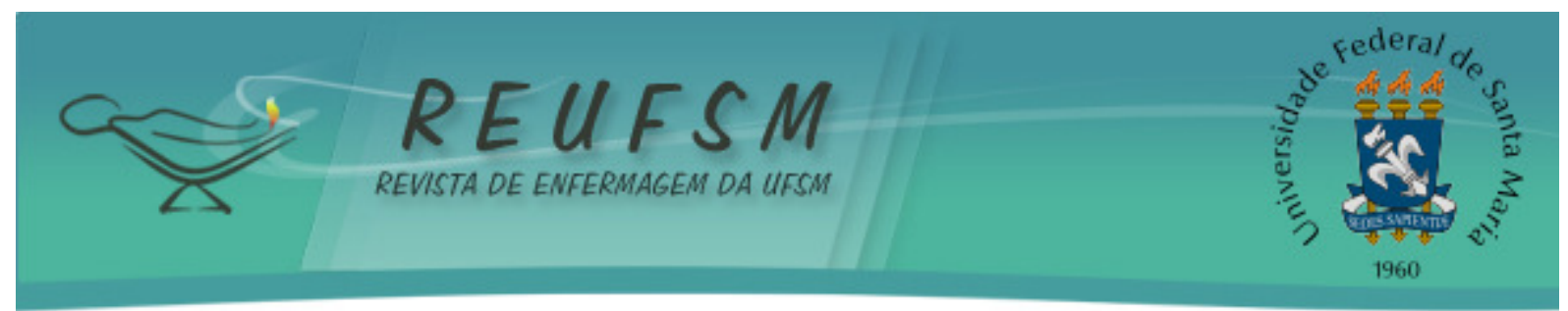

eu procuro aspirar a casa, deixar arejado durante o dia o quartinho dela. Até ursinho de pelúcia, essas coisas, tudo ensacado [...] (FC6)

venho direto pra cá! [serviço de saúde] Não penso duas vezes. Até o pronto socorro eu não vou, porque a gente chega lá, [no hospital] e não é atendido [...] (FC10)

[...] quando dá uma crise de asma é uma coisa terrível, né!? Tu não pode deixar sozinha, tem que ir pro hospital, quando fica muito forte. Porque aí... é só injeção, porque só com o remédio não resolve. Tem que fazer nebulização, tem que ter hora! O cuidado aumenta bastante... [...] (FC13)

A principal meta no tratamento da asma é a obtenção e manutenção do controle da doença, ou seja, o controle das manifestações clínicas e funcionais. Desse modo, as familiares/cuidadoras lançam mão de dispositivos, recursos e adaptação do ambiente físico em prol da preservação da vida da criança. Foi possível observar uma variedade de alternativas para cuidar dos seus filhos no domicílio, de modo que o cuidado desenvolvido pela família, torna-se essencial para mantê-los saudáveis. Isso faz com que em alguns momentos as cuidadoras abram mão de sua vida social e pessoal em função desse cuidado.

Polissemicamente, as familiares retratam essa abnegação em seus discursos:

assim... sabe!? Só que tem que tá [estar]... tem que prestar mais atenção nele, tem que tá [estar] aonde ele tá [está], né!? Não faço mais nada! (FC5)

eu não trabalhava, porque eu só passava no hospital com ele, né!? [...] é diferente, né!? Tu não te lembra de ti, tu só lembra que tu tem que cuidar dele [...] tu acorda a primeira coisa que tu vai fazer é vê [ver] ele. [...] E quando tá [está] doente então... é aquele sofrimento. [...] (FC10)

ah sim, muda tudo! Vive só pra ele, só se envolve com ele. (FC15)

Nesse estudo, os resultados apontam para um cuidado exclusivamente familial, sendo que, geralmente, a mãe é a principal cuidadora da criança com asma. Algumas vezes esse cuidado é solitário e outras vezes é compartilhado com pessoas próximas à família, sem muitos recursos externos para tanto, como pode ser visto nos discursos a seguir:

não, só eu... que cuido! (FC2)

tem a minha sogra, também que me ajuda, quando tô [estou] no serviço. (FC8)

olha, é tudo eu e a minha avó! Não tem... Ela sempre me ajudou. Ela que cuida dele pra eu trabalhar também. \# (FC10)

Polissemicamente, algumas familiares trazem em seus discursos a participação da criança com asma em seu próprio cuidado, conforme as enunciações abaixo: 


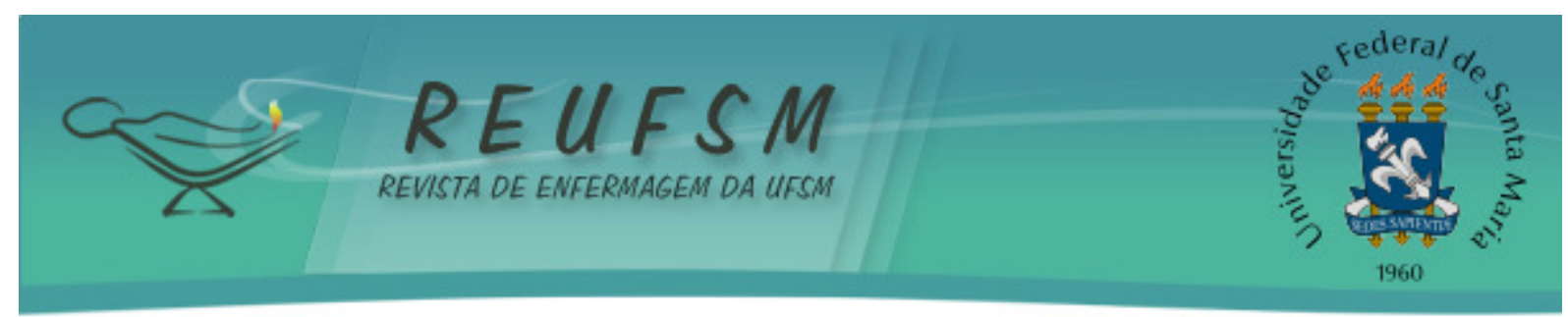

[...] remédio ela que faz de manhã, ela limpa bem o narizinho, é ela mesmo que injeta as gotinhas, ela já aprendeu, então ela faz assim. (FC1)

até ela às vezes vem e diz... "mãe meu remédio, hoje é meu remédio" [parafraseando a filha] Ela pede! [familiar sorri.] (FC11)

[...] ela é uma criança muito obediente, ela adora tomar remédio. Tal hora ela mesmo se antena. [metaforicamente falando] "mãe tá [está] na hora do meu remédio". Então é uma criança que se ajuda [...] (FC 12)

Os discursos mostram a importância de a criança compreender sua doença e conhecer os fatores precipitantes, de modo a contribuir no seu cuidado, evitando o surgimento de novas crises com auxílio das familiares/cuidadoras.

\section{DISCUSSÃO}

As familiares/cuidadoras desenvolvem um cuidado comprometido com a preservação da saúde da criança, a fim de livrá-las dos agravos que possam comprometer a saúde e a qualidade de vida. Porém, para que esse cuidado seja eficiente e em benefício não só da criança, mas de toda a família, os profissionais de saúde devem estar habilitados para oferecer apoio aos familiares. ${ }^{11}$

Nesse sentido, é indispensável que haja uma rede de apoio social que forneça suporte aos familiares/cuidadores. Essa rede deve envolver uma equipe multiprofissional, que necessita ir além dos serviços médicos. ${ }^{12}$

Destaca-se a importância da aproximação do enfermeiro e dos demais profissionais de saúde com a família no processo terapêutico, favorecidos quando assumida uma postura flexível, de escuta ao outro, utilizando uma posição em uma alteridade sem limite, em que a troca de saberes ocorre de forma não horizontal, mas dialógica, considerando-se a demanda do familiar e de sua realidade.

As familiares/cuidadoras utilizam recursos como medicamentos, adequação do ambiente e a busca por serviços de saúde para garantir a sobrevivência de seus filhos. Muitas reorganizam sua rotina a fim de manter um cuidado total à criança, e sacrificam sua vida social e profissional em virtude da fragilidade clínica de seu filho.

Cuidar de uma criança com doença crônica é uma tarefa difícil. Requer articulação entre pessoas e locais inseridos em uma rede social, principalmente no que tange àquelas com asma, que dependem exclusivamente dos cuidados familiares para sobreviver. ${ }^{13}$

0 alto custo com a terapêutica pode interferir na promoção e na adesão ao tratamento e na forma como a família se organiza para o cuidado. Muitas vezes a mesma não possui repertório suficiente para lidar com situações difíceis ou exigências de recursos elevados para manter o tratamento da criança. ${ }^{14}$

As familiares/cuidadoras sentem-se inviabilizadas para buscar auxílio ou acompanhamento da sua própria saúde, abdicando de sua vida social para suprir as necessidades dos filhos e para desenvolver o processo de cuidado. ${ }^{15}$ Muitas vezes, essa condição está atrelada ao suporte social frágil e a dificuldade de acesso aos serviços de saúde. ${ }^{16}$

A adequação no ambiente doméstico é uma das estratégias utilizadas pelas cuidadoras. A higiene do ambiente em que a criança reside é uma das medidas profiláticas recomendadas. Por outro lado, soluções como retirar cortinas, tapetes, ventiladores, condicionadores de ar assim como, algumas vezes, dispensar o animal de estimação, podem frustrar não só a criança, como os irmãos e até mesmo os pais. ${ }^{17}$ 


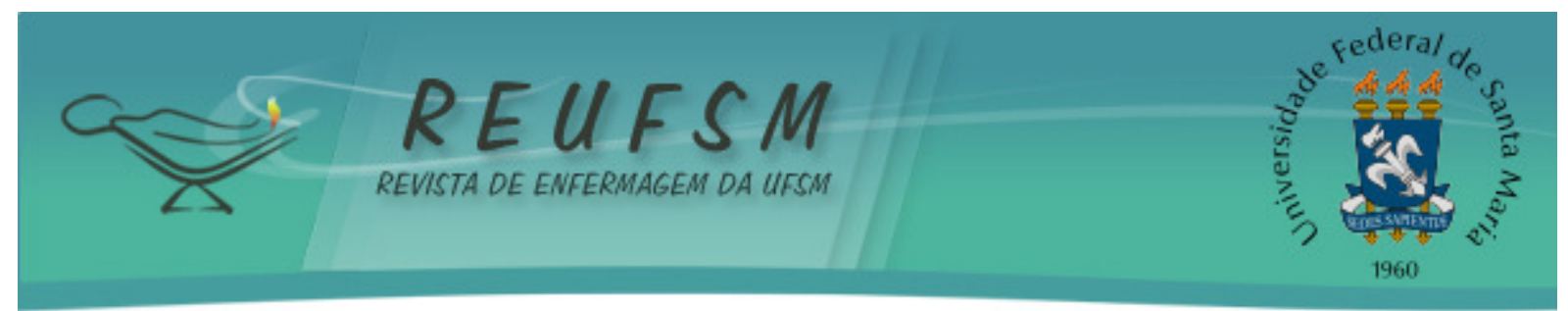

Também aparecem nos discursos providências que as crianças tomam em relação à doença, como o autocuidado que as tornam capazes de tomar iniciativas frente ao seu tratamento. Nesse sentido, é imprescindível que a enfermagem contribua na promoção da saúde, possibilitando a aprendizagem e motivando comportamentos mais independentes. ${ }^{18}$

0 cuidado desenvolvido pelos participantes do estudo, em geral, é realizado pelas mães das crianças com asma, com a ajuda de outras familiares. O círculo familiar da criança envolve os membros mais próximos, e essa rede familiar é essencial para a manutenção do tratamento e a qualidade de vida da criança. As mães, na maioria das vezes, assumem o papel de cuidadoras principais, juntamente com as avós, tias e madrinhas $^{19}$, o que faz com que as mesmas passem a desenvolver estratégias para lidar com os novos contextos e as novas exigências que a vida the impõe. Isso, por sua vez, representa um estímulo para que se sintam mais confiantes para cuidar. ${ }^{1}$

Desse modo, a família possui papel fundamental no cuidado à criança, pois detém a responsabilidade do bem-estar físico, emocional e social de seus membros. Contudo, é no núcleo familiar que a criança tem seu referencial. ${ }^{20}$

Para tanto, faz-se necessário uma estrutura familiar favorável, a fim de evitar o desgaste das cuidadoras provocado pelo cansaço, pelo cuidado intenso e estresse do cotidiano, intrinsecamente relacionados ao cuidado de preservação desenvolvido pela família.

\section{CONSIDERAÇÕES FINAIS}

Conclui-se que as familiares/cuidadoras elencam prioridades nos cuidados à criança com asma no âmbito domiciliar. As mesmas realizam o processo de cuidado voltado à preservação da criança e por conta desse cuidado, muitas vezes, acabam abdicando de sua vida pessoal, social e profissional devido à preocupação com o estado de saúde da criança e com estratégias para manutenção de sua sobrevivência.

Para a familiar/cuidadora o cuidado domiciliar vai além do tratamento, trata-se da conservação da vida da criança, ou seja, um cuidado de preservação. O cuidado é predominantemente desenvolvido pelas mulheres da família e está pautado em estratégias e recursos para a manutenção da sobrevivência dessas crianças.

O cuidado de preservação está pautado na melhoria da saúde e na amenização de sintomas decorrentes da asma. Em alguns casos, é possível identificar que esse cuidado é tão intenso que a criança acaba sendo privada de atividades comuns do seu dia a dia.

O cuidado domiciliar está pautado na experiência feita, pela prática desenvolvida e aperfeiçoada durante os anos, conhecimentos de outros familiares e também nas orientações recebidas pelos profissionais do serviço de saúde, cenário deste estudo.

Embora a asma tenha uma alta incidência na infância, sugere-se a necessidade de estudos que abordem os cuidados domiciliares e as estratégias utilizadas pela família da criança com asma. Recomenda-se que a enfermagem esteja mais próxima das familiares/cuidadoras, tornando o domicílio uma extensão do ambiente de cuidado.

\section{REFERÊNCIAS}

1. Trinca MA, Bicudo IMP, Pelicioni MCF. A interferência da asma no cotidiano das crianças. Rev Bras Crescimento Desenvolv Hum. 2011;21(1):70-84.

2. Brasil. Ministério da Saúde. Secretaria de Atenção Básica. Departamento de Atenção Básica. Doenças respiratórias crônicas [Internet]. Brasília: Ministério da Saúde; 2010 [acesso em 2012 fev 7]. 160 p. Disponível em: 


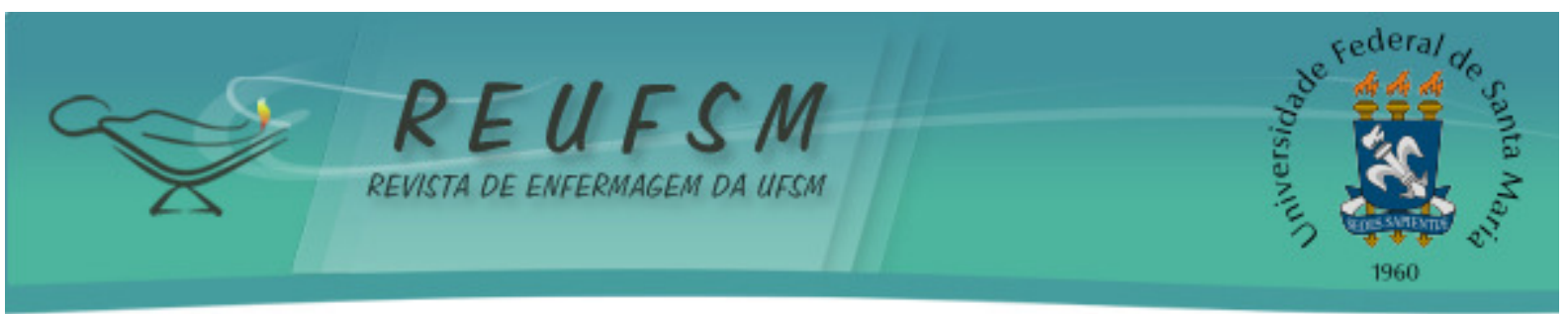

http://bvsms.saude.gov.br/bvs/publicacoes/doencas_respiratorias_cronicas.pdf. (Série A. normas e Manuais Técnicos). (Cadernos de Atenção Básica; 25).

3. Nóbrega VM, Reichert APS, Silva KL, Coutinho SED, Collet N. Impositions and conflicts on the daily routine of families of children with chronic disease. Esc Anna Nery [Internet]. 2012 dez [acesso em 2014 jul 20];16(4):781-8. Disponível em: http: / / www.scielo.br/scielo.php?script=sci_arttext\&pid=S1414-

$81452012000400020 \& \operatorname{lng}=e n \& n r m=i s o \& t$ lng=pt. Doi:10.1590/S1414-81452012000400020.

4. Borba RIH, Ribeiro CA, Ohara CVS, Sarti CA. O mundo da criança portadora de asma grave na escola. Acta Paul Enferm [Internet] 2009 mar [acesso em 2014 out 2];22(N Esp):921-7. Disponível em: http://www.scielo.br/pdf/ape/v22nspe/15.pdf. Doi: 10.1590/S0103-21002009000700015.

5. Neves ET, Cabral IE, Silveira A. Rede familial de crianças com necessidades especiais de saúde: implicações para a enfermagem. Rev Latinoam Enferm [Internet]. 2013 abr [acesso em 2014 jul 19];21(2):[9 telas]. Disponível em: http://www.scielo.br/pdf/rlae/v21n2/pt_0104-1169-rlae-21-02-0562.pdf.

Doi:10.1590/S0104-11692013000200013.

6. Neves ET, Silveira A. Desafios para os cuidadores familiares de crianças com necessidades especiais de saúde: contribuições da enfermagem. Rev Enferm UFPE Online [Internet]. 2013 [acesso 2015 fev 10];7(5):1458-62.Disponível em: http://www.revista.ufpe.br/revistaenfermagem/index.php/revista/article/download/322 9/6147.

7. Minayo MS. O desafio do conhecimento: pesquisa qualitativa em saúde. 12 ed. São Paulo: Hucitec; 2010.

8. Denzin N, Lincoln Y. The sage handbook of qualitative research. 3th ed. California/USA: SAGE publications; 2005. 1210 p.

9. Orlandi EP. Análise de discurso: princípios e procedimentos. $7^{\mathrm{a}}$ ed. Campinas: Pontes; 2009.

10. Brasil. Ministério da Saúde. Conselho Nacional de Saúde. Resolução CNS No 466, de 12 de dezembro de 2012. Aprova as diretrizes e normas regulamentadoras de pesquisas envolvendo seres humanos. Diário Oficial da União, Brasília; 2013 jun 13. Seção 1, p. 59.

11. Silva MDB, Silva LR, Meneses IMS. O cuidado materno no manejo da asma infantilcontribuição da enfermagem transcultural. Esc Anna Nery [Internet]. 2009 dez [acesso em 2014 set 20];13(4):772-9. Disponível em: http: / / www.scielo.br/scielo.php?script=sci_arttext\&pid=S1414-81452009000400012. Doi: 10.1590/S1414-81452009000400012.

12. Miller AR, Condin CJ, McKellin WH, Shaw N, Klassen AF, Sheps S. Continuity of care for children with complex chronic health conditions: parents' perspectives. BMC Health Serv Res. 2009;9:242-52.

13. Moraes JRMM, Cabral IE. The social network of children with special healthcare needs in the (in) visibility of nursing care. Rev Latinoam Enferm [Internet]. 2012 [acesso em 2014 fev 10];20(2):282-8. Disponível em: http: / /www.scielo.br/scielo.php?script=sci_arttext\&pid=S0104-11692012000200010.

Doi:10.1590/S0104-11692012000200010. 


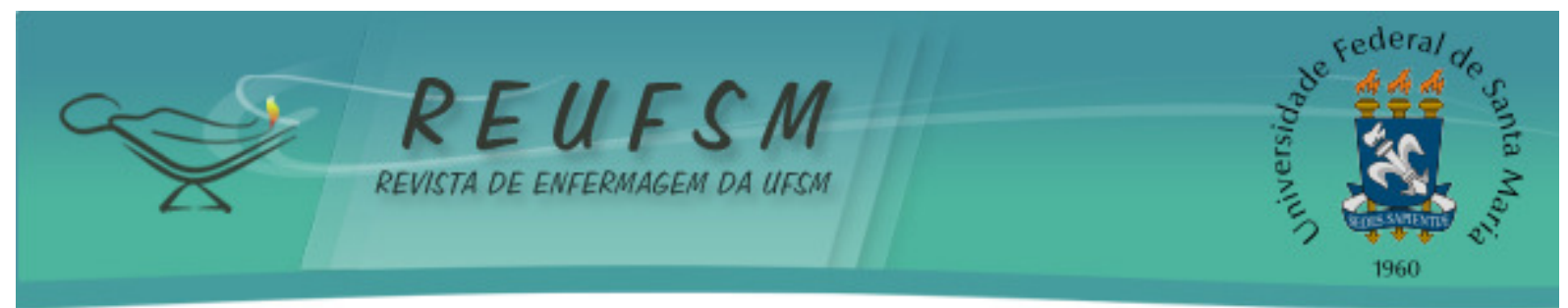

14. Mendonça MB, Ferreira EP. Adesão ao tratamento da asma na infância: dificuldades enfrentadas por cuidadoras. Rev Bras Cresc Desenv Hum. 2005;15(1):56-68.

15. Silveira A, Neves ET, Paula CC. Cuidado familial das crianças com necessidades especiais de saúde: um processo (sobre) natural e de (super) proteção. Texto \& Contexto Enferm [Internet]. $2013 \mathrm{dez}$ [acesso em 2014 set 13];22(4):1106-14. Disponível em: http: //www.scielo.br/scielo.php?script=sci_arttext\&pid=S0104-07072013000400029. Doi: 10.1590/S0104-07072013000400029.

16. Porterfield SL, McBride TD. The effect of poverty and caregiver education on perceived need and access to health services among children with special health care needs. Am J Public Health [Internet]. 2007 [acesso em 2014 mar 27];97(2):323-9. Disponível em: http://www.ncbi.nlm.nih.gov/pmc/articles/PMC1781389/.

17. Bosi DR, Reis AOA. A criança asmática na família: estudo de uma representação. Rev Bras Crescimento Desenvolv Hum. 2000;10(2):60-79.

18. Ferraz L, Almeida FM, Girardi F, Soares SC. Assistência de enfermagem na promoção do autocuidado aos portadores de necessidades especiais. Rev Enferm UERJ. 2007;15(4):597600.

19. Neves ET, Andres B, Silveira A, Arrué AM. A rede social de cuidados de uma criança com necessidade especial de saúde. Rev Eletrônica Enferm [Internet]. 2013 jun [acesso em 2014 abr 12];15(2):533-40. Disponível em: https://www.fen.ufg.br/fen_revista/v15/n2/pdf/v15n2a28.pdf. Doi: doi: 10.5216/ree.v15i2.17064.

20. Comaru NRC, Monteiro ARM. O cuidado domiciliar à criança em quimioterapia na perspectiva do cuidador familiar. Rev Gaúcha Enferm [Internet]. 2008 set [acesso em 2014 maio 3];29(3):423-30. Disponível em: http://seer.ufrgs.br/RevistaGauchadeEnfermagem/article/view/6770.

Data de recebimento: $12 / 10 / 2014$

Data de aceite: 06/07/2015

Contato do autor responsável: Camila Fernandes Wild

Endereço postal: Avenida Nossa Senhora das Dores, $n^{\circ} 768$, apt 202, Bairro Dores. Santa Maria, RS, Brasil.

E-mail: camilinhah_wild@hotmail.com 